Article

\title{
Determination of the Material Parameters in the Holzapfel-Gasser-Ogden Constitutive Model for Simulation of Age-Dependent Material Nonlinear Behavior for Aortic Wall Tissue under Uniaxial Tension
}

\author{
Up Huh ${ }^{1,2}$, Chung-Won Lee ${ }^{1,2}$, Ji-Hun You ${ }^{3}$, Chan-Hee Song ${ }^{3}$, Chi-Seung Lee ${ }^{2,3,4, *(D)}$ and \\ Dong-Man Ryu ${ }^{2,3, *(1)}$ \\ 1 Department of Thoracic and Cardiovascular Surgery, School of Medicine, Pusan National University, \\ Busan 49241, Korea \\ 2 Biomedical Research Institute, Pusan National University Hospital, Busan 49241, Korea \\ 3 Department of Biomedical Engineering, School of Medicine, Pusan National University, Busan 49241, Korea \\ 4 Department of Convergence Medicine, School of Medicine, Pusan National University, Busan 49241, Korea \\ * Correspondence: victorich@pusan.ac.kr (C.-S.L.); babopanda@pusan.ac.kr (D.-M.R.); \\ Tel.: +82-51-240-6867 (C.-S.L.)
}

Received: 9 May 2019; Accepted: 13 July 2019; Published: 17 July 2019

check for updates

\begin{abstract}
In this study, computational simulations and experiments were performed to investigate the mechanical behavior of the aorta wall because of the increasing occurrences of aorta-related diseases. The study focused on the deformation and strength of porcine and healthy human abdominal aortic tissues under uniaxial tensile loading. The experiments for the mechanical behavior of the arterial tissue were conducted using a uniaxial tensile test apparatus to validate the simulation results. In addition, the strength and stretching of the tissues in the abdominal aorta of a healthy human as a function of age were investigated based on the uniaxial tensile tests. Moreover, computational simulations using the ABAQUS finite element analysis program were conducted on the experimental scenarios based on age, and the Holzapfel-Gasser-Ogden (HGO) model was applied during the simulation. The material parameters and formulae to be used in the HGO model were proposed to identify the failure stress and stretch correlation with age.
\end{abstract}

Keywords: abdominal aorta; uniaxial tensile test; material nonlinear behavior; finite element analysis

\section{Introduction}

The aorta is the primary artery through which blood leaves the human heart before it flows through the entire human body, and it is typically divided into several sections based on anatomical source, as shown in Figure 1 [1-4]. Based on the anatomical compartment, it is categorized as the thoracic aorta from the heart to the diaphragm and the abdominal aorta from the diaphragm to the bifurcation of the aorta, respectively. Another classification method classifies the aorta according to its route and blood flow direction. Hence, the aorta goes out from the heart and down as the ascending aorta and then performs a hairpin turn. Following this, the aorta runs as the descending aorta, which is divided into two major parts: thoracic aorta and abdominal aorta. The aorta ends at a bifurcation, which is divided into two main blood vessels: the common iliac arteries and a smaller midline vessel, the median sacral artery [5]. 


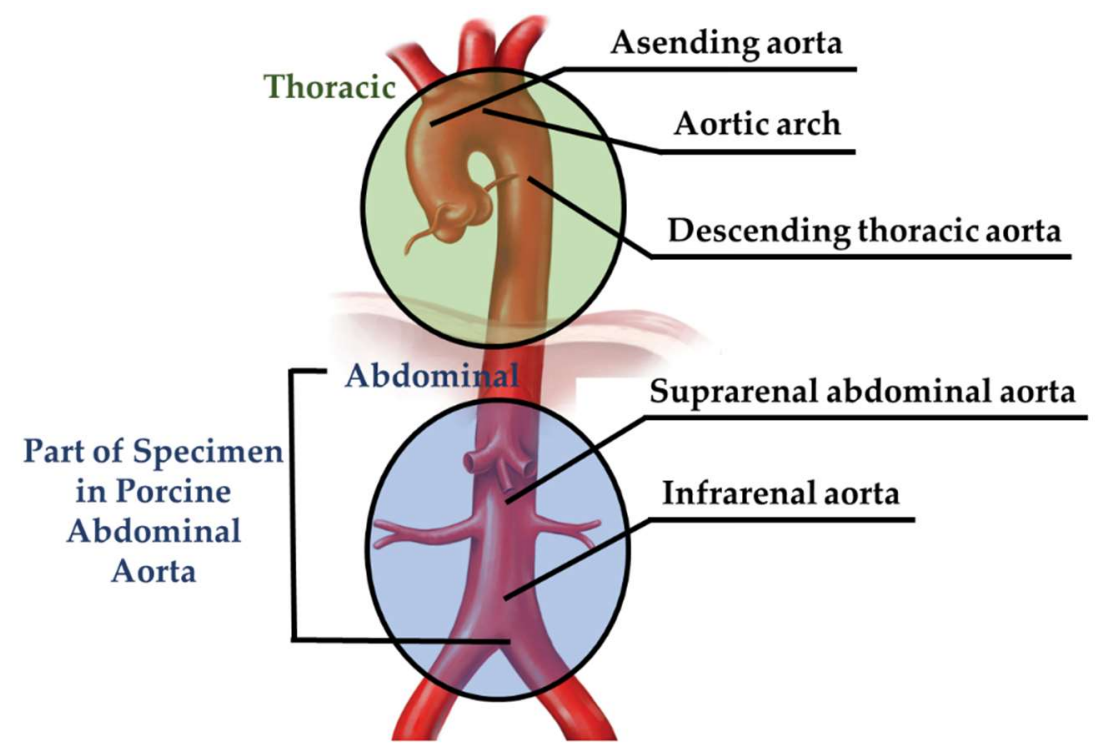

Figure 1. Schematic of the aorta and its segments.

The aorta exhibits the characteristics of a hyper-elastic and viscoelastic material. It is composed of a heterogeneous mixture of smooth muscle nerves, endothelial cells, intimal cells, fibroblast-like cells, and a complex extracellular matrix. The arterial wall consists of three layers, intima, media, and adventitia. In particular, the smooth muscles and extracellular matrix are the largest components of the arterial wall in the media layer. Specifically, the media layer of the arterial wall consists of concentric elastic lamella, namely smooth muscles and an elastic matrix. The smooth muscle component does not transform the diameter of the aorta significantly; instead, it serves to increase the stiffness and viscoelasticity of the aortic wall when activated. The elastic matrix controls the biomechanical properties of the aorta and forms lamellae consisting of elastic fibers, collagens, proteoglycans, and glycosaminoglycans [6]. Thus, the vascular smooth muscle cells can make significant contributions to aortic stiffness, and many studies have been conducted on them. In particular, Morgan et al. [7,8] measured the mouse aortic mechanical properties in order to assess the vascular smooth muscle cell component of stiffness in the aortic wall according to age. In addition, they examined the mechanical properties of blood vessels across multiple length scales and identified the focal adhesion of the vascular smooth muscle cells. Meininger et al. [9] determined whether vascular smooth muscle cells adhesion to type I collagen was transformed in parallel with the changes in the vascular smooth muscle cells' contractile state induced by vasoconstrictors and vasodilators, and they proposed that the signal transduction pathways modulating vascular smooth muscle cell contractile activation and relaxation, as well as extracellular matrix adhesion interact during regulation of the contractile state. Leloup et al. [10] investigated whether basal nitric oxide activity and voltage-gated $\mathrm{Ca}^{2+}$ channel-mediated contractions differed between the smaller, muscular arteries (femoral and mesenteric arteries) and the larger, elastic conduit vessels (aorta and carotid artery) of mice. As a result, they assumed that the different physiological behavior of elastic and muscular arteries of young adults were linked to the well-known observation that arterial stiffness develops differently with aging.

In addition to studies on vascular smooth muscle cells, studies on the properties of the aorta and diseases such as aortic aneurysm and aortic dissection have been conducted in the past few decades [11-19]. In particular, Yang et al. [15] conducted biomechanical experiments on the porcine abdominal artery by uniaxial elongation and relaxation tests in both the circumferential and longitudinal directions and applied a combined logarithm and polynomial strain energy equation to model the elastic response of the specimens. The reduced relaxation function was modified by integrating a rational equation as a corrective factor to simulate the strain-dependent relaxation effects accurately. Horny et al. [16] proposed a regression model capable of estimating the pre-strain of a human abdominal 
aorta. A database including the length and diameter of the abdominal aorta, heart weight, thickness of the left ventricle, and degree of atherosclerosis was obtained by evaluating 156 male cadavers. In the database, a significant correlation between the age, pre-strain, diameter, and atherosclerosis was identified, which was best fitted to a power law equation. Ninomiya et al. [17] studied the biomechanical failure properties and histological composition of a human non-aneurysmal aorta. They extracted the material specimens from 26 human cadavers and measured the failure stress, tension, and strain in circumferentially-oriented strips. Courtial et al. [18] defined the biomechanical parameters of a healthy human abdominal aorta and developed materials for aortic phantom production. These phantoms used in the training of endovascular treatment must exhibit the same morphology and mechanical behavior properties as those of the aorta of the patient. Therefore, they conducted ex vivo experiments by uniaxial tensile and dynamic simple shear testing of six healthy human abdominal aortas to confirm the biomechanical parameters accurately. Laksari et al. [19] examined the in vitro anisotropic mechanical behavior of canine aortic tissue and focused on the spatial variations in its properties in the axis direction of the vessel. Hence, uniaxial stretch tests on canine aortic specimens in the circumferential and longitudinal directions, as well as histological examinations to identify the fiber orientations of the tissues were performed. In addition, they examined a constitutive model that contained both phenomenological and structural elements to consider the macroscopic and microstructural behaviors of the tissue.

Further, precise constitutive models of soft biological tissue coupled with appropriate numerical approaches have been continuously developed from these experimental results. In other words, it has been developed as a phenomenological approach that describes the macroscopic characteristic of a material as a continuum in order to simulate the mechanical behavior including the stiffness and direction of fibers of aortic material [20-23]. Although such studies were conducted, they were not sufficiently large in scale to study the effects of the age range, the numerical model applied for material nonlinear damage modeling, and the criterion for rupture of the aortic tissue. Therefore, in this study, the numerical simulation approach was suggested to simulate material properties according to age. In the step for determining the material properties, the uniaxial tensile tests were conducted on porcine aortic specimens in the circumferential and longitudinal directions, and the numerical simulations that the Holzapfel-Gasser-Ogden (HGO) model [22-24] was applied to simulate the behavior of the arterial tissue were performed using the ABAQUS finite element program. The results between experiment and numerical simulation were compared to determine the reliability of the simulation. In addition, the experimental results for the healthy human abdominal aorta specimens were investigated and analyzed based on age, and the material constants associated with the elastic modulus, stress, and strain in the numerical model were estimated from the numerical simulations according to age. From these results, the correlation between age and material constants was examined, and the formulae for estimating material constants based on age were proposed.

\section{Constitutive Model}

In industrial and technological applications, many materials exhibit an anisotropic elastic behavior because of the fiber orientation, such as fiber-reinforced composites, reinforced rubber, wood, arterial wall, and heart tissue. In particular, they present nonlinear elastic and anisotropic characteristics under large deformations because of the rearrangements in the microstructure, such as the reorientation of the fiber directions with deformation. Hence, the simulation of these nonlinear effects requires constitutive models formulated within the framework of anisotropic hyper-elasticity [25].

Hyper-elastic materials are expressed in terms of the strain energy potential, which defines the strain energy stored in the material per unit of the reference volume as a function of the deformation at that point in the material. Strain-based and invariant-based formulas are used to express the strain energy potential of anisotropic hyper-elastic materials. Theories related to these methods have been applied in computational biomechanics [20-24,26-28]. In particular, the Fung [20] and HGO models [22-24] have been used widely in various simulations of the arterial wall and heart tissue. In the Fung [20] 
model, the underlying assumption is based on a strain-based formulation in that the preferred material directions are initially aligned with an orthogonal coordinate system in the reference configuration. These directions may become non-orthogonal only after deformation. Concurrently, in the HGO model [22-24], unlike in the strain-based formula, the fiber orientation in the invariant-based formula does not need to be orthogonal in the initial configuration. Therefore, the authors proposed a constitutive law for the description of the mechanical response of arterial tissue and considered the arterial wall as a thin-walled cylindrical tube consisting of layers.

\subsection{Strain-Based Formula}

The strain energy function is described as a component of a suitable strain tensor, such as the Green strain tensor:

$$
U=U\left(\varepsilon^{G}\right)
$$

where $\varepsilon^{G}=\frac{1}{2}(\mathbf{C}-\mathbf{I})$ is the Green strain tensor, $\mathbf{C}=\mathbf{F}^{T} \mathbf{F}$ is the right Cauchy-Green deformation tensor, $\mathbf{F}$ is the deformation gradient, which is expressed in terms of the displacement vector, $\mathbf{u}(\mathbf{F}=\nabla \mathbf{u}+\mathbf{I})$, and $\mathbf{I}$ is the identity matrix. Without loss of generality, the strain energy function can be written as follows:

$$
U=U\left(\bar{\varepsilon}^{G}, J\right)
$$

where $\bar{\varepsilon}^{G}=\frac{1}{2}(\overline{\mathbf{C}}-\mathbf{I})$ is the modified Green strain tensor, $\bar{C}=J^{-\frac{2}{3}} \mathbf{C}$ is the modified right Cauchy-Green strain, and $J=\operatorname{det} \mathbf{F}$. The fundamental hypothesis in the above models based on the strain-based formula is that the preferred material directions are initially arranged with an orthogonal coordinate system in the reference stress-free configuration. These directions may become non-orthogonal only after deformation [20].

From Equation (2), the variation in $U$ is given as follows:

$$
\delta U=\frac{\partial U}{\partial \bar{\varepsilon}^{G}}: \delta \bar{\varepsilon}^{G}+\frac{\partial U}{\partial J} \delta J=\overline{\mathbf{F}} \frac{\partial U}{\partial \bar{\varepsilon}^{G}} \overline{\mathbf{F}}^{\mathrm{T}}: \delta \mathbf{e}+\frac{\partial U}{\partial J} \delta J
$$

Using the principle of virtual work, the variation in the strain energy potential can be written as follows:

$$
\delta W_{I}=\int_{V^{0}} J\left(\mathbf{S}: \delta \mathbf{e}-p \delta \varepsilon^{\mathrm{vol}}\right) d V^{0}=\int_{V^{0}} \delta U d V^{0}
$$

For a compressible material, the strain variations are arbitrary; hence, this equation defines the stress components for such a material as follows:

$$
\begin{gathered}
\mathbf{S}=\frac{1}{J} \operatorname{DEV}\left[\overline{\mathbf{F}} \frac{\partial U}{\partial \bar{\varepsilon}^{G}} \overline{\mathbf{F}}^{T}\right] \\
p=-\frac{\partial U}{\partial J}
\end{gathered}
$$

With the pure displacement formulation from which the strain invariants are calculated, the kinematic variables of a finite element (FE) model could behave insufficiently if the material is incompressible. The problem is that the stiffness matrix is almost singular from a numerical perspective since the effective bulk modulus of a material is larger than the effective shear modulus. Hence, this causes difficulties in obtaining the solution of the discretized equilibrium equation.

\subsection{Invariant-Based Formula}

From the continuum theory of fiber-reinforced composites [27], the strain energy function could be expressed as an invariant deformation tensor and a fiber orientation. For example, consider a composite material composed of an isotropic hyper-elastic matrix reinforced with fibers. In the reference configuration, 
the orientation of a fiber is characterized by a set of unit vectors. Assuming that the strain energy depends on the fiber orientation, as well as the deformation, it can be expressed as follows:

$$
U=U\left(\mathbf{C}, \mathbf{A}_{\alpha}\right) ; \alpha=1, \ldots, N
$$

The strain energy of the material should not change when both the matrix and fibers in the reference configuration are rigid-body rotations. Subsequently, the strain energy can be expressed as an isotropic function of a non-reducible set of scalar invariants formed based on tensor $\mathbf{C}$ and vectors $\mathbf{A}_{\alpha}$ :

$$
U=U\left(\bar{I}_{1}, \bar{I}_{2}, J, \bar{I}_{4(\alpha \beta)}, \bar{I}_{5(\alpha \beta)}, \zeta_{\alpha \beta}\right) ; \alpha=1, \ldots, N ; \beta=1, \ldots, \alpha
$$

where $\bar{I}_{1}$ and $\bar{I}_{2}$ are the first and second deviatoric strain invariants; $\bar{I}_{4(\alpha \beta)}$ and $\bar{I}_{5(\alpha \beta)}$ are the pseudo-invariants of $\overline{\mathbf{C}}, \mathbf{A}_{\alpha}$ and $\mathbf{A}_{\beta}$; and $J$ is the third strain invariant, as follows:

$$
\bar{I}_{4(\alpha \beta)}=\mathbf{A}_{\alpha} \overline{\mathbf{C}} \mathbf{A}_{\beta} \text {, and } \bar{I}_{5(\alpha \beta)}=\mathbf{A}_{\alpha} \overline{\mathbf{C}}^{2} \mathbf{A}_{\beta} ; \alpha=1, \ldots, N ; \beta=1, \ldots, \alpha
$$

On the other hand, $\zeta_{\alpha \beta}$ is a geometric constant, such as the angular cosine between the fiber orientations in the reference configuration, namely,

$$
\zeta_{\alpha \beta}=\mathbf{A}_{\alpha} \mathbf{A}_{\beta} ; \alpha=1, \ldots, N ; \beta=1, \ldots, \alpha
$$

In the invariant-based formulation, the fiber orientations need not be orthogonal in the initial configuration [22-24]. From Equation (8), the variation in $U$ is expressed as follows:

$$
\delta U=\frac{\partial U}{\partial \bar{I}_{1}} \delta \bar{I}_{1}+\frac{\partial U}{\partial \bar{I}_{2}} \delta \bar{I}_{2}+\frac{\partial U}{\partial J} \delta J+\sum_{\alpha=1}^{N} \sum_{\beta=1}^{\alpha}\left(\frac{\partial U}{\partial \bar{I}_{4(\alpha \beta)}} \delta \bar{I}_{4(\alpha \beta)}+\frac{\partial U}{\partial \bar{I}_{5(\alpha \beta)}} \delta \bar{I}_{5(\alpha \beta)}\right)
$$

From the principle of virtual work, Equation (4), the stress components for a compressible material can be expressed as follows:

$$
\begin{gathered}
\mathbf{S}=\frac{2}{J} \operatorname{DEV}\left[\left(\frac{\partial U}{\partial \bar{I}_{1}}+\bar{I}_{1} \frac{\partial U}{\partial \bar{I}_{2}}\right) \overline{\mathbf{B}}-\frac{\partial U}{\partial \bar{I}_{2}} \overline{\mathbf{B B}}\right]+\sum_{\alpha=1}^{N} \sum_{\beta=1}^{\alpha} \frac{\partial U}{\partial \bar{I}_{4(\alpha \beta)}} \operatorname{DEV}\left(\overline{\mathbf{a}}_{\alpha} \overline{\mathbf{a}}_{\beta}+\overline{\mathbf{a}}_{\beta} \overline{\mathbf{a}}_{\alpha}\right) \\
+\sum_{\alpha=1}^{N} \sum_{\beta=1}^{\alpha} \frac{\partial U}{\partial \bar{I}_{5(\alpha \beta)}} \operatorname{DEV}\left(\overline{\mathbf{a}}_{\alpha} \overline{\mathbf{a}}_{\beta}^{\prime}+\overline{\mathbf{a}}_{\alpha}^{\prime} \overline{\mathbf{a}}_{\beta}+\overline{\mathbf{a}}_{\beta} \overline{\mathbf{a}}_{\alpha}^{\prime}+\overline{\mathbf{a}}_{\beta}^{\prime} \overline{\mathbf{a}}_{\alpha}\right) \\
p=-\frac{\partial U}{\partial J}
\end{gathered}
$$

where $\overline{\mathbf{a}}_{\alpha} \overline{\mathbf{F}} \mathbf{A}_{\alpha}$ and $\overline{\mathbf{a}}_{\alpha}^{\prime} \overline{\mathbf{B}} \mathbf{a}_{\alpha}$.

\subsection{Anisotropic Hyper-Elastic Materials}

The form of the strain energy potential for modeling arterial layers with distributed collagen fiber orientations is based on that proposed by Holzapfel et al. [22-24]:

$$
\begin{aligned}
U=C_{10}\left(\bar{I}_{1}-3\right)+ & \frac{1}{D}\left(\frac{\left(J^{e l}\right)^{2}-1}{2}-\ln J^{e l}\right)+\frac{k_{1}}{2 k_{2}} \sum_{\alpha=1}^{N}\left\{\exp \left[k_{2}\left\langle\bar{E}_{\alpha}\right\rangle^{2}\right]-1\right\} \\
& \bar{E}_{\alpha} \kappa\left(\bar{I}_{1}-3\right)+(1-3 \kappa)\left(\bar{I}_{4(\alpha \alpha)}-1\right)
\end{aligned}
$$

where $U$ is the strain energy per unit of the reference volume; $C_{10}, D, k_{1}, k_{2}$, and $\kappa$ are temperature-dependent material parameters; $N$ is the number of families of fibers $(N \leq 3)$; $\bar{I}_{1}$ is the first invariant of $\overline{\mathbf{C}} ; j^{e l}$ is the elastic volume ratio; and $\bar{I}_{4(\alpha \alpha)}$ are the pseudo-invariants of $\overline{\mathbf{C}}$ and $\mathbf{A}_{\alpha}$. 
This model presumes that the orientation of the collagen fibers in each family is distributed rotationally symmetrically with respect to the mean preferred orientation. The parameter, $\kappa(0 \leq \kappa \leq 1 / 3)$, expresses the level of dispersion in the fiber directions. If $\rho(\Theta)$ is the orientation density function that identifies the distribution (it implies the normalized number of fibers with orientations in the range $[\Theta, \Theta+d \Theta]$ associated with the mean orientation) [24], then the parameter, $\kappa$, is defined as follows:

$$
\kappa=\frac{1}{4} \int_{0}^{\pi} \rho(\Theta) \sin ^{3} \Theta d \Theta .
$$

It is also presumed that all the fiber groups exhibit the same mechanical properties and the same dispersion. When $\kappa=0$, the fibers are completely aligned (no dispersion). When $\kappa=1 / 3$, the fibers are randomly distributed, and the material becomes isotropic. This corresponds to the spherical orientation density function. The strain-like quantity, $\bar{E}_{\alpha}$, identifies the deformation of the fiber group with the mean direction, $\mathbf{A}_{\alpha} \cdot \bar{E}_{\alpha}=\bar{I}_{4(\alpha \alpha)}-1$ for completely aligned fibers $(\kappa=0)$, and $\bar{E}_{\alpha}=\left(\bar{I}_{1}-3\right) / 3$ for randomly-distributed fibers $(\kappa=1 / 3)$.

The first two terms in the strain energy function express the distortional and volumetric contributions of the non-collagenous isotropic ground material. The third term expresses the contributions from other families of collagen fibers, considering the effects of dispersion. The basic presumption of the model is that collagen fibers can support only tension because they would buckle under compressive loads. Hence, the anisotropic contribution in the strain energy function only appears when the strain of the fibers is positive, or equivalently, when $\bar{E}_{\alpha}>0$. This condition is enforced by the term $\left\langle\bar{E}_{\alpha}\right\rangle$, where operator $\langle\cdot\rangle$ represents the Macaulay bracket and is defined as $\langle x\rangle=1 / 2(|x|+x)$ [25].

The initial deviatoric elasticity tensor, $\overline{\mathbf{D}}_{0}$, and bulk modulus, $K_{0}$, are given by:

$$
\begin{gathered}
\overline{\mathbf{D}}_{0}=4 C_{10} \mathfrak{I}+2(1-3 \kappa)^{2} k_{1} \sum_{\alpha=1}^{N} H\left(\bar{E}_{\alpha}\right) \mathbf{A}_{\alpha} \mathbf{A}_{\alpha} \mathbf{A}_{\alpha} \mathbf{A}_{\alpha} \\
K_{0}=\frac{2}{D}
\end{gathered}
$$

where $\mathfrak{I}$ is the fourth-order unit tensor and $H(x)$ is the Heaviside unit step function.

\section{Experiments and Simulations}

This research was approved by the Institutional Animal Care and Use Committees (IACUC) of the Pusan National University Yangsan Hospital (PNUYH). The approval number is PNUYH-2018-065.

\subsection{Experiment Details for the Material Test}

For uniaxial tensile test, ten tissue specimens according to the loading direction were extracted from five porcine abdominal aortic tissues. After extraction, the specimens were immediately placed in warm saline, and then, the uniaxial tensile tests were conducted within $12 \mathrm{~h}$ of harvest. In order to obtain robust rectangular strips (15-mm width, 25-mm length, and 10-mm width in the middle), the specimens were sliced in the circumferential and longitudinal directions of the aorta [29], as presented in Table 1. The width and thickness for the specimens were measured at three locations, and the average data were adopted. The specimens were mounted in the uniaxial tensile test apparatus and continuously wetted with saline [11]. Figure 2 shows the schematic of the universal testing machine and tensile load directions, which are subdivided into circumferential and longitudinal directions, relative to the axis of the aorta. Moreover, in the uniaxial tensile test, the ends of the specimens were fixed using sand paper and a fixing apparatus, which maintained the tissues in place by a zig.

The behaviors of the arterial tissues with the loading direction were investigated with a 500.0-N EZ-TEST load cell, and the experimental results were utilized to verify the numerical model. For the tests, a universal testing machine (EZ-TEST 500 N, Shimadzu, Busan, South Korea) was adopted. 
Table 1. Tissue specimens of the porcine abdominal aorta in the tensile test (AC: Aorta and Circumferentially (Load direction), AL: Aorta and Longitudinally (Load direction)).

\begin{tabular}{cccccc}
\hline \multirow{2}{*}{ Specimen } & \multicolumn{3}{c}{ Sample Dimension (in mm) } & \multirow{2}{*}{$\begin{array}{c}\text { Tensile Load Direction } \\
\text { Relative to Oriented Strip }\end{array}$} \\
\cline { 2 - 4 } & Width (Top) & Width (Middle) & Length & Thickness & \\
\hline AC1 & 14.96 & 9.98 & 24.97 & 1.98 & Circumferentially \\
AC2 & 15.10 & 10.04 & 25.05 & 2.01 & Circumferentially \\
AC3 & 14.98 & 10.01 & 25.03 & 2.01 & Circumferentially \\
AC4 & 15.05 & 10.01 & 25.01 & 1.99 & Circumferentially \\
AC5 & 15.01 & 9.99 & 25.00 & 2.03 & Circumferentially \\
AL1 & 15.02 & 10.02 & 24.98 & 2.01 & Longitudinally \\
AL2 & 15.04 & 10.02 & 24.99 & 2.02 & Longitudinally \\
AL3 & 14.97 & 10.03 & 25.01 & 1.97 & Longitudinally \\
AL4 & 14.99 & 9.98 & 24.98 & 2.01 & Longitudinally \\
AL5 & 15.04 & 9.97 & 25.03 & 2.01 & Longitudinally \\
\hline
\end{tabular}

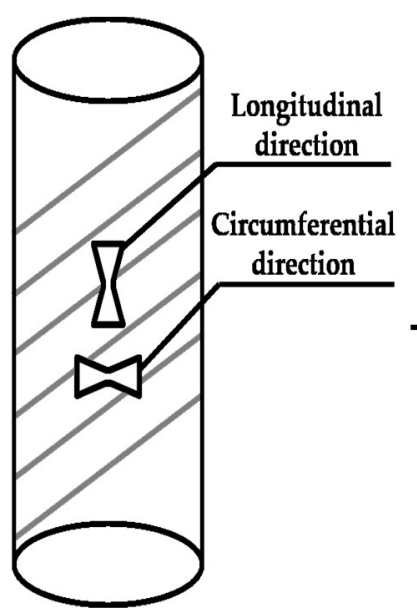

(a)
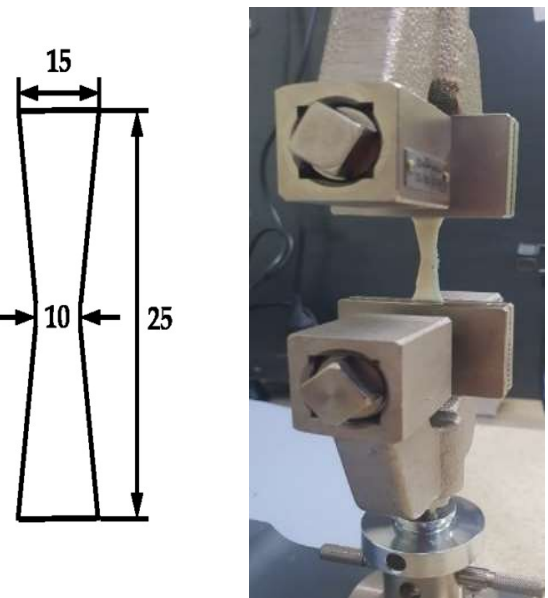

(b)

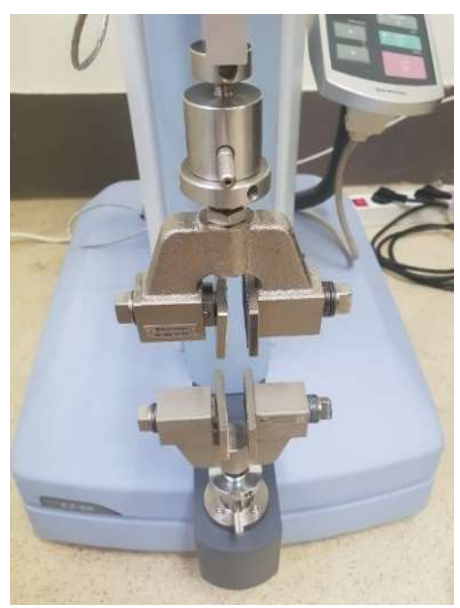

(c)

Figure 2. Schematic of the uniaxial tensile test specimens and experimental apparatus. (a) Dimensions of the test specimens; dimensions in millimeters. (b) Closer view of the specimen fixation. (c) Universal testing machine.

The preconditioning test was adopted to guarantee the straight configuration of the test specimen before the main test. In addition, the viscoelastic effect was not considered during the main test. In other words, a preconditioning test, involving 10 loading and unloading cycles of the specimen strip to $5 \%$ of its length at $4 \%$ of specimen length $/ \mathrm{min}(1 \mathrm{~mm} / \mathrm{min})$, was conducted. After preconditioning, the strip was uniaxially extended at $4 \%$ specimen length $/ \mathrm{min}(1 \mathrm{~mm} / \mathrm{min})$ until failure.

\subsection{Simulation Details for the Material Test}

One of the well-established commercial finite element analysis programs, ABAQUS, was used for simulation of the uniaxial tensile test. The material parameters in the HGO model were estimated based on the stress-stretch curve of the porcine abdominal aortic tissue as a function of the tissue orientation to validate the computational simulation technique. The geometries of the uniaxial tensile test specimens used were constructed by considering the dimensions in the experiment and reference [17], as shown in Figure 3a. The orientations for the collagen fiber in the intimal, medial, and adventitial strips were set as $18.8^{\circ}, 37.8^{\circ}$, and $58.9^{\circ}$, respectively, as shown in Figure $3 b$ [26] and Figure 4.

As indicated in Section 3.1, fixed and straight-line motion conditions were applied as the experimental boundary and loading conditions, as shown in Figure 3. In particular, under the loading condition, the tensile loading directions, which were divided into circumferential and longitudinal directions relative 
to the axis of the aorta, were considered. In addition, the HGO model for an anisotropic hyper-elastic material was applied for determining the material properties in the simulation.

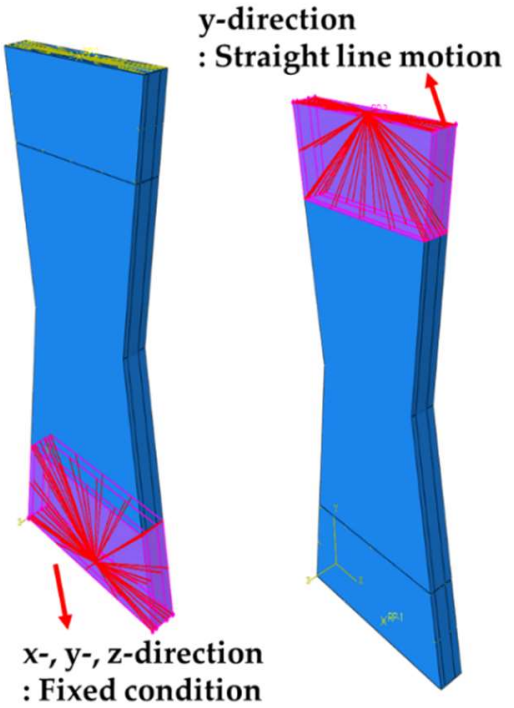

(a)

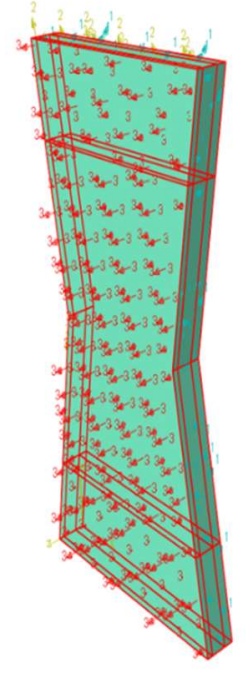

(b)

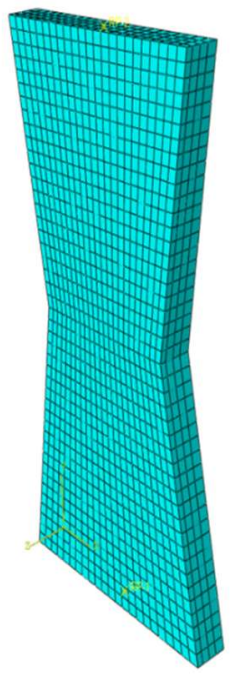

Figure 3. (a) Boundary and loading conditions and (b) orientations of the fiber and mesh in the tensile test specimen.
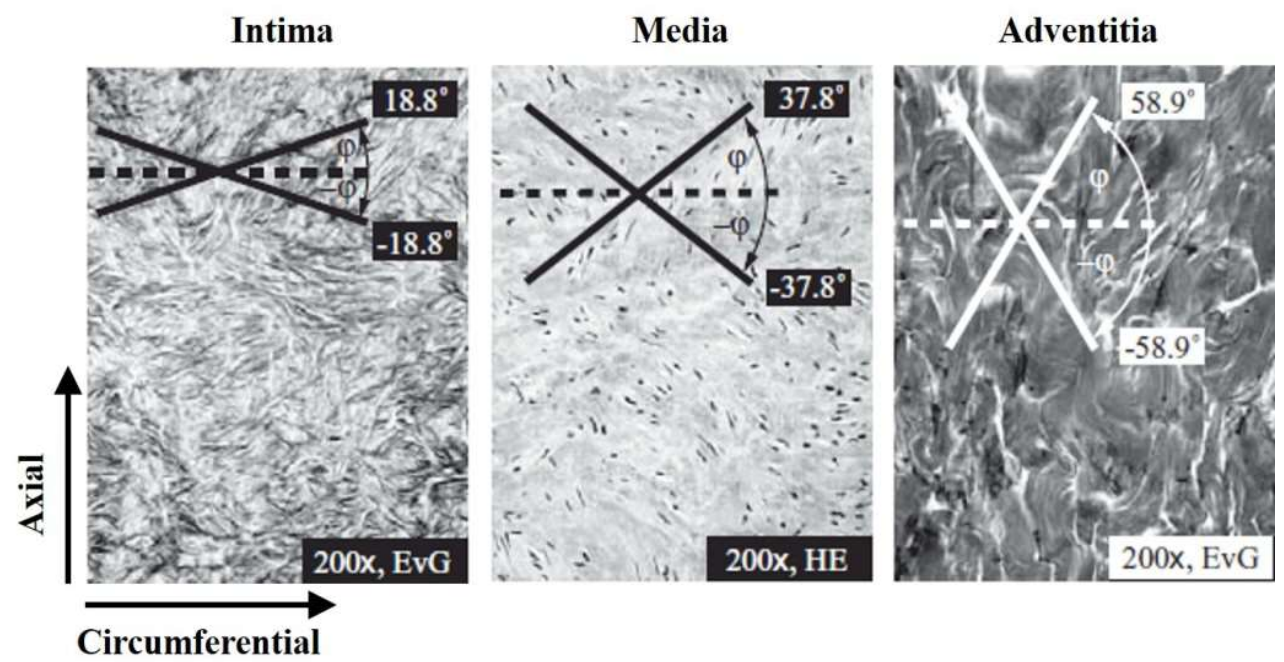

Figure 4. Circumferential orientation of the collagen fiber in the layer [26].

The hexahedral (C3D8RH-type) element, which is the eight-node linear brick, reduced integration with hourglass control, hybrid with constant pressure, was adopted during simulation. When the material response is incompressible such as soft tissue, the solution to a problem cannot be obtained in terms of the displacement history only, since a purely hydrostatic pressure can be added without changing the displacement. The nearly incompressible case showed behavior approaching this limit, in that a very small change in displacement produced extremely large changes in pressure, so that a purely displacement-based solution was too sensitive to be useful numerically. Accordingly, ABAQUS removed this singular behavior in the system by treating the pressure stress as an independently interpolated basic solution variable, coupled to the displacement solution through the constitutive theory and the compatibility condition, with this coupling implemented by a Lagrange multiplier. This independent interpolation of pressure stress was the basis of these "hybrid" elements. More 
precisely, they were "mixed formulation" elements, using a mixture of displacement and stress variables with an augmented variational principle to approximate the equilibrium equations and compatibility conditions [25].

The hybrid elements also remedied the problem of volume strain "locking," which can occur at much lower values of Poisson's ratio. Volume strain locking occurs if the finite element mesh cannot properly represent incompressible deformations. Volume strain locking can be avoided in regular displacement elements by fully- or selectively reduced integration [25].

For this reason, in most hyperelastic analyses for incompressible material using ABAQUS, such as rubber, polymer, and soft tissue, the C3D8RH element is adopted. Hence, the C3D8RH element was adopted during simulation in this study.

The mesh sensitivity check was also carried out prior to simulation as shown in Figure 5. As shown in this figure, the Cauchy stress was stabilized when the total number of elements was more than approximately 3000 . Accordingly, the optimal number of elements in the specimen was determined to be 4048 .

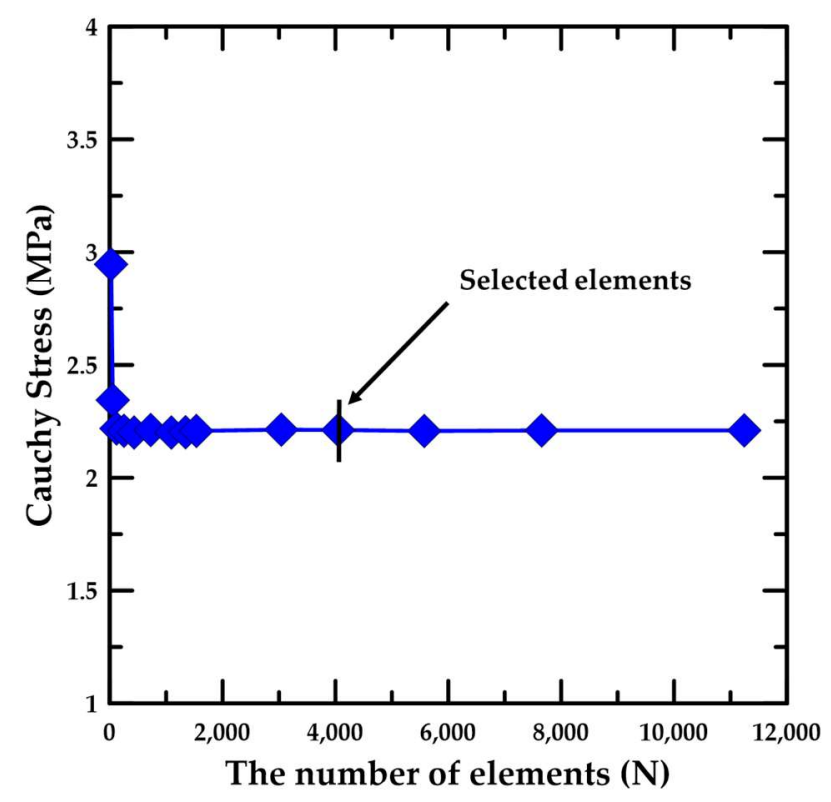

Figure 5. Results of the mesh size convergence study for the specimen.

\subsection{Results for the Material Test}

It was necessary to vZalidate the computational simulation results by comparing with the experimental results to ensure their reliability. Therefore, in this study, the material parameters of the HGO model [24] were adjusted with a simulation test based on the stress-stretch curve of the tissues in the porcine abdominal aorta. In addition, computational simulations and uniaxial tensile tests of circumferentially- and longitudinally-oriented strips were conducted. Consequently, the stress-stretch curves were calculated from the simulation results, and the results were compared with the experimental results, as shown in Figure 6.

The correlation between the simulation result and average value of the experimental results demonstrated a maximum error rate (1-(experimental results/simulation results)) of 0.0193 and 0.0945 for the circumferentially- and longitudinally-oriented strips, respectively. In addition, as shown from the experimental results, the aortic tissue exhibited different tensile stresses and stretches based on the fiber orientation because its three layers had different fiber directions. In particular, based on the average values of the experimental results, the circumferentially-oriented strip sample exhibited a maximum tensile strength of $2.49 \mathrm{MPa}$, which was $18.3 \%$ higher than the maximum value for the longitudinally-oriented strip sample. Concurrently, from the stretch results, the longitudinally-oriented strip sample exhibited a maximum tensile stretch value of 1.63 , which was $7.7 \%$ higher than the 
maximum value of the circumferentially-oriented strip sample. It appeared that the aortic tissue exhibited different tensile characteristics based on the fiber orientation. Moreover, the material constants for the fiber orientation were confirmed from the parametric simulation, as shown in Figure 6.

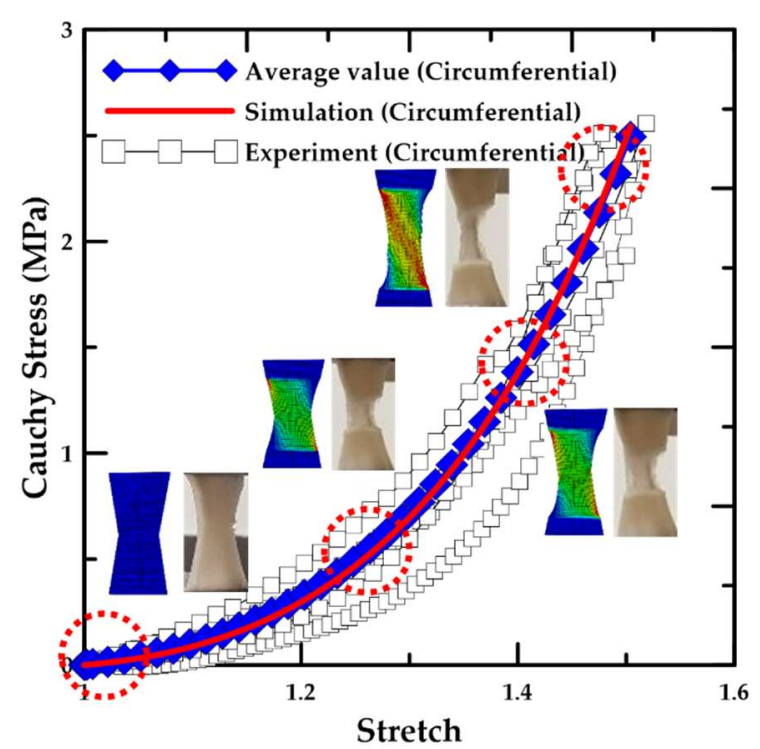

(a)

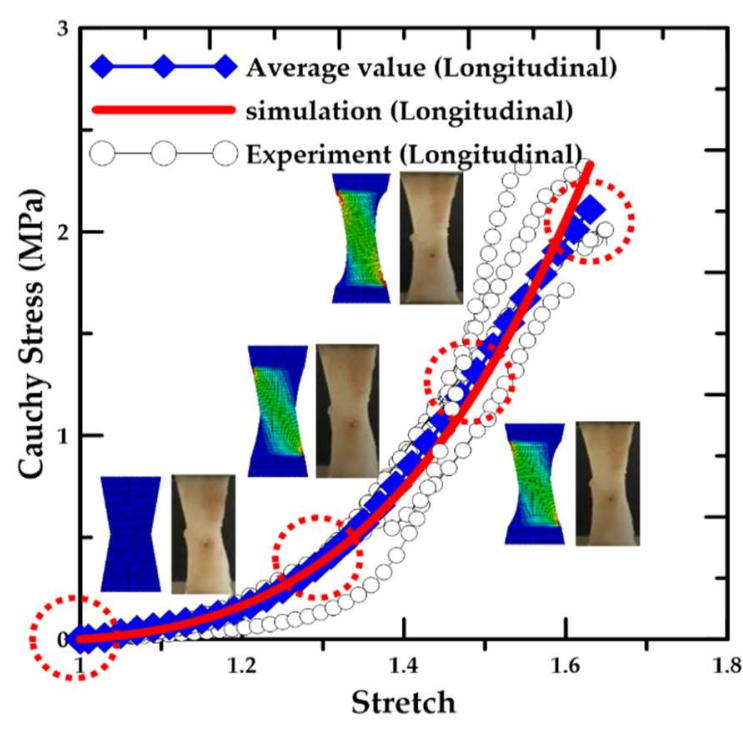

(b)

Figure 6. Stress-stretch curves of the porcine arterial tissue according to the loading direction determined from the tensile tests and simulations on (a) circumferentially- and (b) longitudinally-oriented strips.

\section{Results}

\subsection{Experiment Details and Trend Lines}

In this study, aortic material properties were investigated from $40-80 \mathrm{~s}$ to establish a basis for simulating aortic disease and the experimental results based on age obtained from the uniaxial tensile tests performed by Ninomiya et al. [17]. The tissue specimens in the healthy human abdominal aorta were extracted from cadavers. The specimens were sectioned circumferentially in the aorta and obtained as rectangular strips $(4 \mathrm{~mm} \times 40 \mathrm{~mm}$, width $\times$ length). Figure 7 shows the experimental results for the failure stress and stretch obtained from the aortic tissue specimens of humans aged 46-89 years when subjected to a uniaxial tensile load on the MPa scale. It is clear that the samples exhibited failure stresses from approximately $0.42 \mathrm{MPa}-2.32 \mathrm{MPa}$ and failure stretch values from approximately $1.24-1.73$, and it can be seen that the number of stress and stretch values was slightly different because even if the tissue specimens had different stress values, they had similar stretch values.

In addition, in order to calculate the trend line, the exponential regression method, which is a simple nonlinear statistical processing method, was applied based on these experimental results, as shown in Figure 7. The exponential regression equation is as follows.

$$
\begin{gathered}
y=a e^{b x}, \\
\ln y=\ln \left(a e^{b x}\right), \\
\ln y=\ln a+b x,
\end{gathered}
$$

where the coefficients, $a$ and $b$, are defined as follows.

$$
b=\frac{n \sum_{i=1}^{n}\left(\ln y_{i} \times x_{i}\right)-\sum_{i=1}^{n}\left(\ln y_{i}\right) \sum_{i=1}^{n}\left(x_{i}\right)}{n \sum_{i=1}^{n}\left(x_{i}^{2}\right)-\left(\sum_{i=1}^{n}\left(x_{i}\right)\right)^{2}},
$$




$$
\ln a=\frac{\sum_{i=1}^{n}\left(\ln y_{i}\right)}{n}-b \frac{\sum_{i=1}^{n}\left(x_{i}\right)}{n}
$$

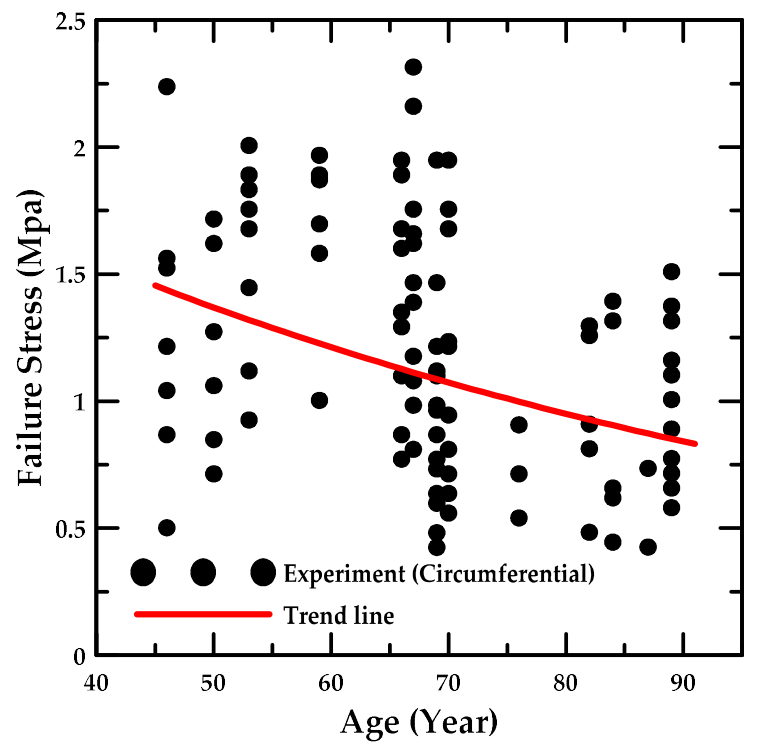

(a)

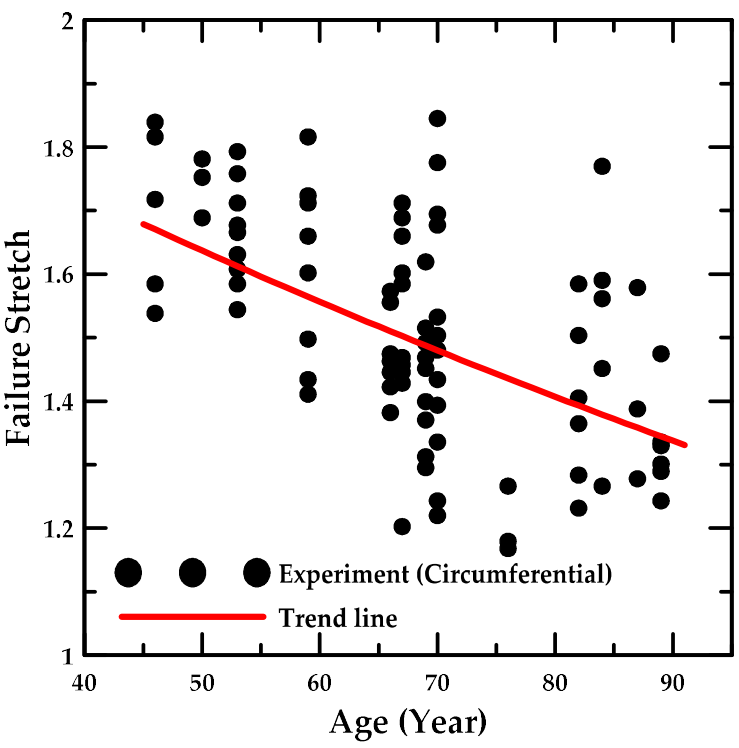

(b)

Figure 7. Failure (a) stress and (b) stretch distribution as a function of age.

The procedure for calculating the trend line was to display a scatter diagram based on the constructed database and to assess an appropriate regression model from the transformation for scale of each axis. Therefore, in this study, the exponential regression was selected, and the logarithm was taken on both sides as shown in Equations (19)-(21), while the coefficients were calculated from Equations (22)-(23). It appeared that the failure stress and stretch in the aortic tissue affected the decrease in the tensile strength significantly; as the age of the specimen increases, the trend for the database could be recognized. In addition, the minimum and maximum values of the trend were determined, and the criteria for the damage characteristics of the aortic tissue in a healthy human abdominal aorta could be established from these trend lines.

\subsection{Simulation Results Based on Age}

In this study, the two lines of the maximum and minimum values were estimated based on the trend line of the average values and experimental results for the healthy human abdominal aorta specimens, and the trend lines had deviations of $44.7 \%$ and $-44.7 \%$, and $11.9 \%$ and $-11.9 \%$ in the failure stress and stretch graph, respectively, as shown in Figure 8. In addition, the numerical simulations for the tensile test were performed from 46 years- 89 years based on the failure stress and stretch in the two trend lines of the maximum and minimum values. In the numerical simulation, the material parameters, $k_{1}$ and $k_{2}$, according to age were estimated based on the failure stress and stretch because the parameters were associated with the stress and strain of soft tissue. The material parameter, $C_{10}$, according to age was estimated by comparing the stress-stretch curves obtained from the numerical simulation, the porcine experiment, and the reference for the experiment of the aorta $[12,15,16,19,29,30]$. These simulation results obtained from estimating the material parameters according to age were compared with the trend lines of the maximum and minimum values, and it was clear that the maximum error rates in the trend lines of the maximum and minimum values were 0.0013 and 0.0082 , respectively. In addition, according to the line graph, the average failure stress and stretch of the healthy human abdominal aorta specimens decreased from $1.44 \mathrm{MPa}-0.85 \mathrm{MPa}$ and from 1.67-1.34, respectively, as the age increased from 46 years- 89 years. The trend line ranges of the failure stress and stretch at the age of 46 years were $0.79-2.09 \mathrm{MPa}$ and $1.47-1.87$, respectively. At the age of 89 years, the trend line ranges 
of the failure stress and stretch were $0.20-1.50$ and $1.14-1.54$, which were $28.2-74.7 \%$ and $17.6-22.4 \%$ lower than the results at the age of 46 years, respectively.

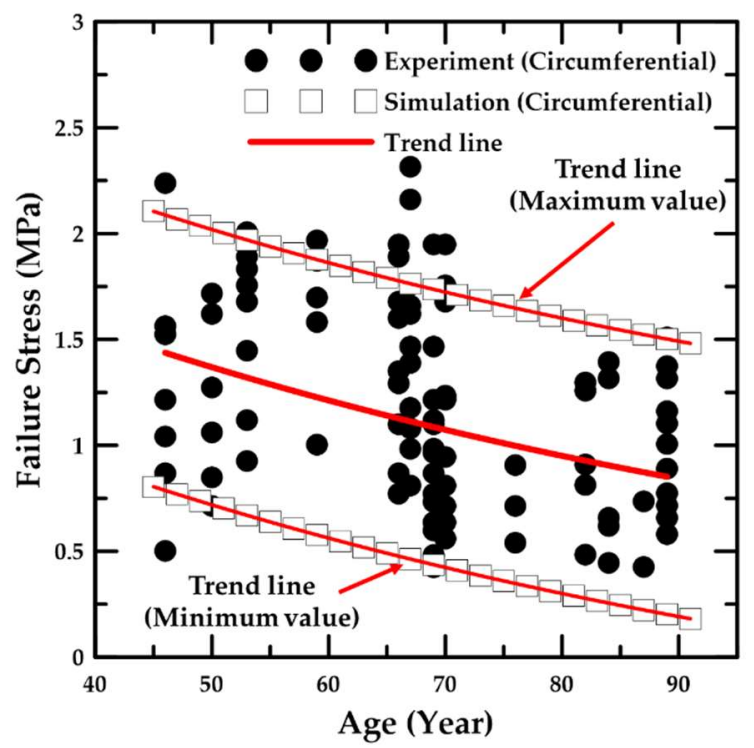

(a)

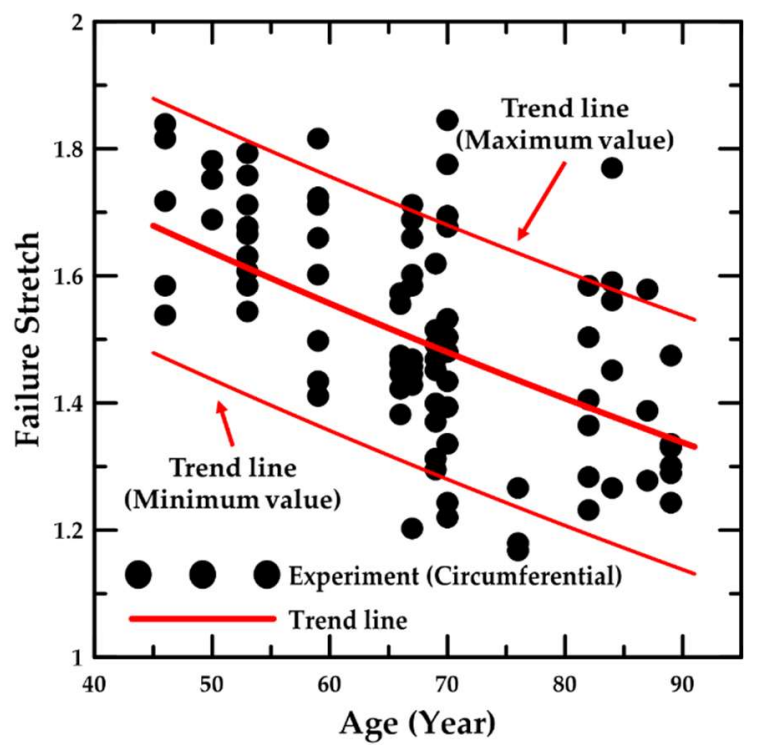

(b)

Figure 8. Experimental and simulation results; trend lines for (a) failure stress and (b) stretch.

The line graph presented in Figure 9 compares the uniaxial tensile strength of abdominal aortic tissue samples for two different loading directions on the megapascal scale for ages 46-89 years. In this simulation, the same material constants were used according to the loading direction. It was found that the trend line range of the failure stress at the age of 46 years was $0.59-2.02 \mathrm{MPa}$ for the longitudinal oriented strips, which was 35.6-79.7\% higher than that at the age of 89 years. In particular, it was clear that the failure stress on the circumferentially-oriented strips at ages 46-89 years was $0.07-0.22 \mathrm{MPa}$ higher than that on the longitudinally-oriented strips. This may be owed to the different orientations of the collagen fiber in the aortic tissue.

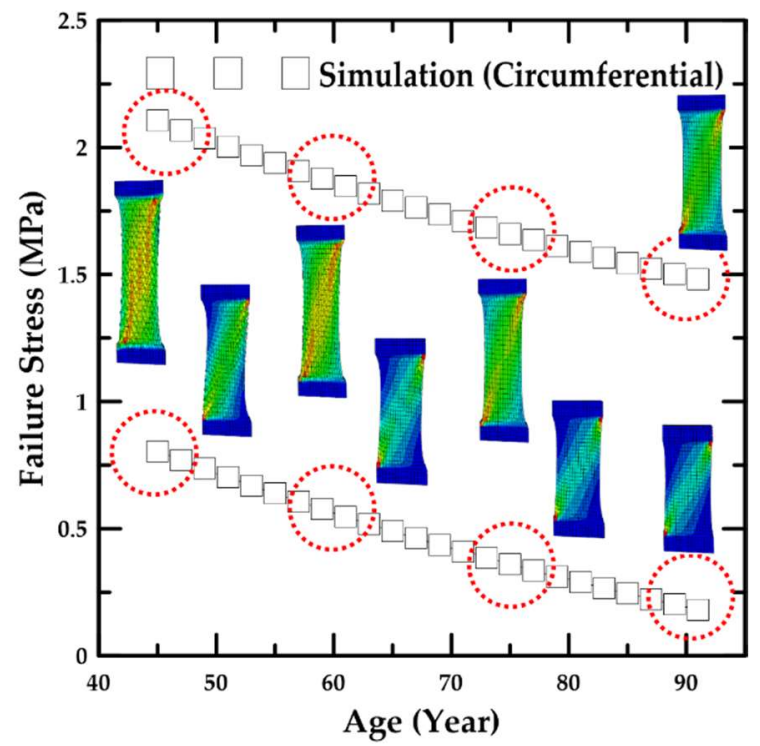

(a)

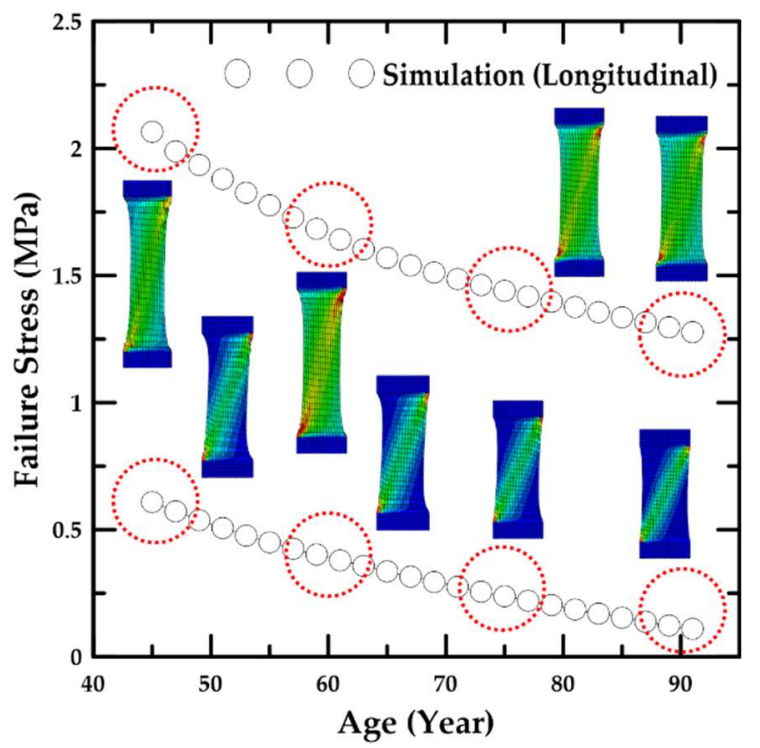

(b)

Figure 9. Simulation results under tensile loading along (a) circumferential and (b) longitudinal directions. 


\subsection{Parametric Study for the Material Constants of the HGO Model}

In this parametric study, the material parameters in the HGO model [22], namely, $C_{10}, k_{1}$, and $k_{2}$, which are associated with the Young's modulus, stress, and strain of the material, were examined from the results based on the age and loading direction, as shown in Figure 10. Generally, the maximum and minimum trend lines of the material parameters increased consistently for the ages of 46-89 years. According to the line graph, the values of the material parameter, $C_{10}$, increased from $0.190-0.233$ and from $0.001-0.005$ on the maximum and minimum trend lines, respectively. Moreover, the values of the material parameter, $k_{1}$, increased nonlinearly from $0.081-0.282$ and from $1.040-8.050$ on the maximum and minimum trend lines, respectively. The values of the material parameter, $k_{2}$, also increased nonlinearly from 2.530-6.486 and from 8.4-72.0 on the maximum and minimum trend lines, respectively. In particular, for the results for above 78 years old, the value of the material parameter, $k_{2}$, on the minimum trend line increased rapidly from 35-72. This is attributable to the samples attaining the failure stress at lower stretch values as the age increased.

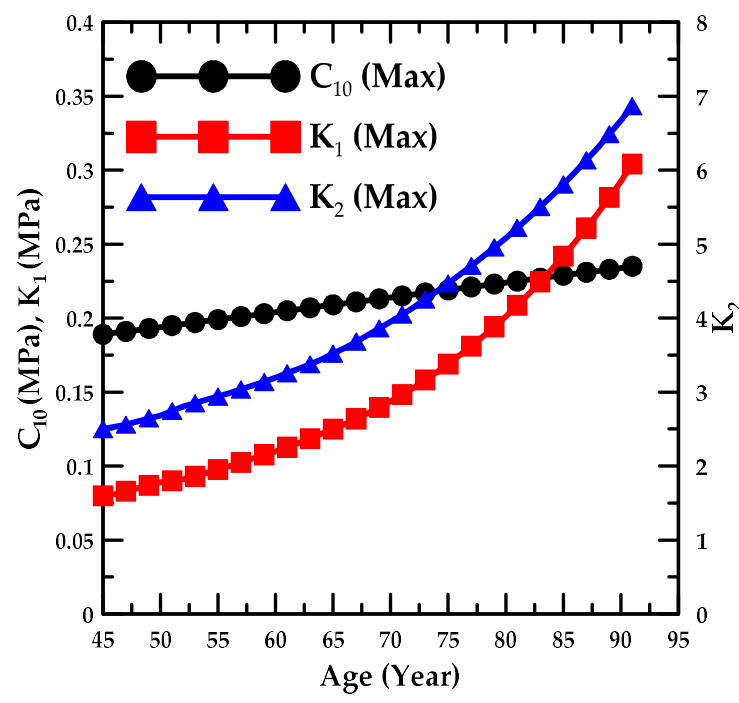

(a)

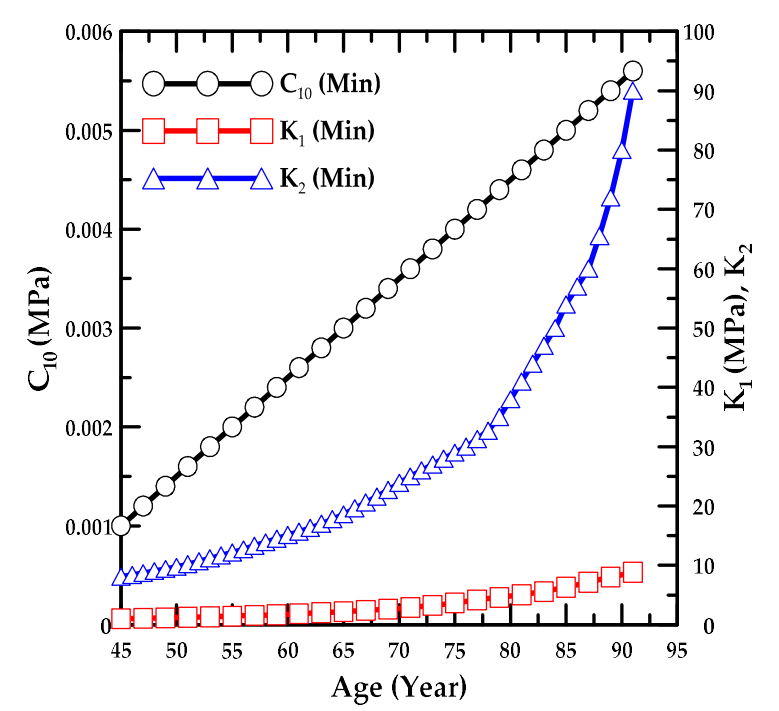

(b)

Figure 10. Variation in the material constants as a function of age in the trend line range ((a) maximum and (b) minimum).

In addition, in this parametric study, the formulas for calculating the values of the material parameters in the HGO model [22] based on age $(N)$ were investigated, which are expressed in Equations (24)-(29) and shown in Figure 11.

$$
\begin{gathered}
C_{10, \text { max }}=\alpha_{1, \text { max }} \times N+\alpha_{2, \text { max }} \\
C_{10, \text { min }}=\alpha_{1, \text { min }} \times N-\alpha_{2, \text { min }} \\
k_{1, \text { max }}=\beta_{1, \text { max }} \times N^{4}+\beta_{2, \text { max }} \times N^{3}+\beta_{3, \text { max }} \times N^{2}+\beta_{4, \text { max }} \times N+\beta_{5, \text { max }} \\
k_{1, \text { min }}=\beta_{1, \text { min }} \times N^{4}+\beta_{2, \text { min }} \times N^{3}+\beta_{3, \text { min }} \times N^{2}+\beta_{4, \text { min }} \times N+\beta_{5, \text { min }} \\
k_{2, \text { max }}=\gamma_{1, \text { max }} \times N^{4}+\gamma_{2, \text { max }} \times N^{3}+\gamma_{3, \text { max }} \times N^{2}+\gamma_{4, \text { max }} \times N+\gamma_{5, \text { max }} \\
k_{2, \text { min }}=\gamma_{1, \text { min }} \times N^{5}+\gamma_{2, \text { min }} \times N^{4}+\gamma_{3, \text { min }} \times N^{3}+\gamma_{4, \text { min }} \times N^{2}+\gamma_{5, \text { min }} \times N+\gamma_{6, \text { min }}
\end{gathered}
$$

Table 2 provides the values of the coefficients and R-squared of the material parameters in Equations (24)-(29). The R-squared implies the proportion of the variance in a dependent variable that can be distinguished from an independent variable. Therefore, according to the values in Table 2, 
the R-squared ranged from $0.9988-1.0$ in the correlation between the values of the material constant and formulae.

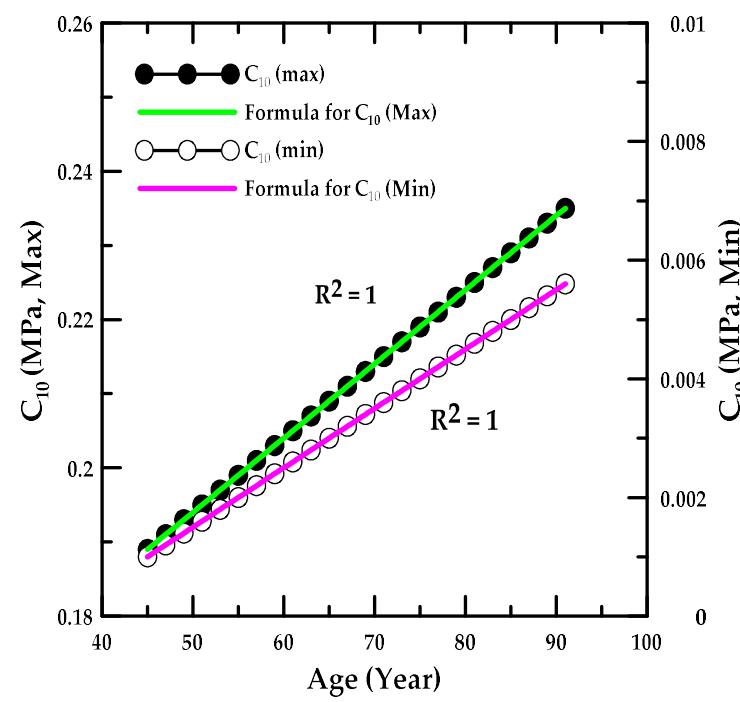

(a)

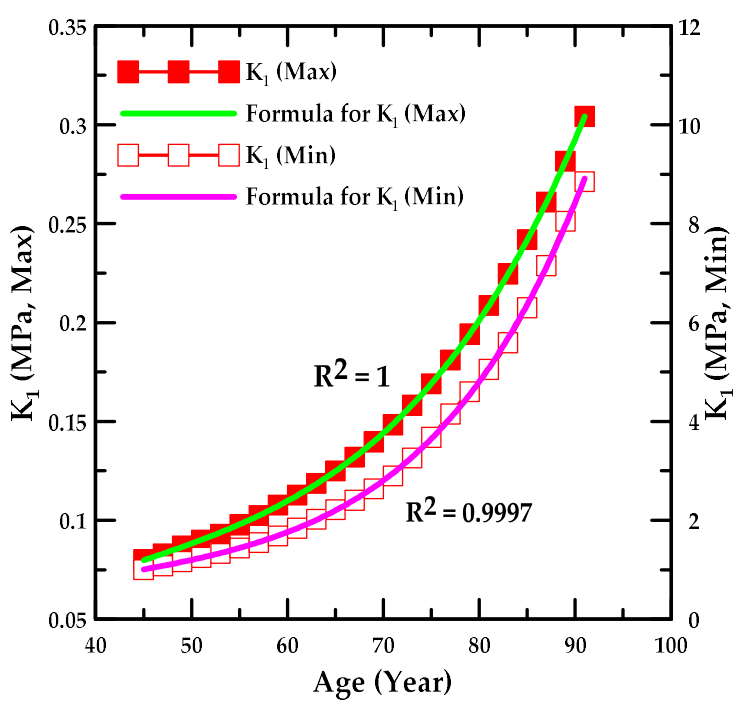

(b)

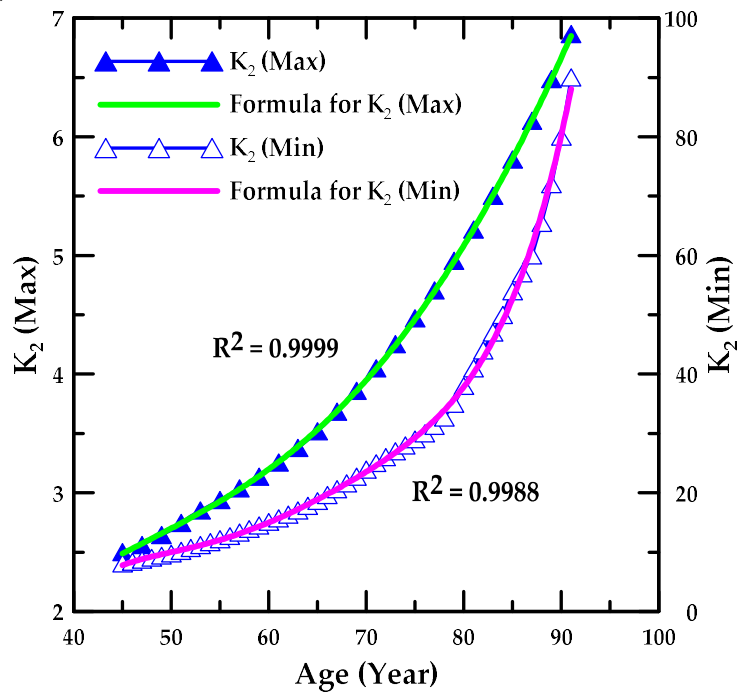

(c)

Figure 11. Comparison between material parameters $\left((\mathbf{a}) C_{10},(\mathbf{b}) k_{1}\right.$, and (c) $\left.k_{2}\right)$ and formulae based on age.

Table 2. Coefficient and R-squared in the correlation between the values of the material parameter and formulae.

\begin{tabular}{|c|c|c|c|c|c|c|}
\hline Material Constant & \multicolumn{2}{|c|}{$\alpha_{1}$} & \multicolumn{2}{|c|}{$\alpha_{2}$} & \multicolumn{2}{|c|}{$\mathbf{R}^{2}$} \\
\hline $\mathrm{C}_{10, \max }$ & \multicolumn{2}{|c|}{0.001} & \multicolumn{2}{|c|}{0.144} & \multicolumn{2}{|c|}{1.0} \\
\hline $\mathrm{C}_{10, \min }$ & \multicolumn{2}{|c|}{0.0001} & \multicolumn{2}{|c|}{0.0035} & \multicolumn{2}{|c|}{1.0} \\
\hline Material Constant & $\beta_{1}, \gamma_{1}$ & $\beta_{2}, \gamma_{2}$ & $\beta_{3}, \gamma_{3}$ & $\beta_{4}, \gamma_{4}$ & $\beta_{5}, \gamma_{5}, \gamma_{6}$ & $\mathbf{R}^{2}$ \\
\hline $\mathrm{k}_{1, \max }$ & $8.26 \times 10^{-9}$ & $-5.11 \times 10^{-7}$ & $-2.30 \times 10^{-5}$ & 0.0038 & -0.0315 & 1.0 \\
\hline $\mathrm{k}_{1, \text { min }}$ & $8.58 \times 10^{-7}$ & -0.0001 & 0.0099 & -0.2863 & 3.6079 & 0.9997 \\
\hline $\mathrm{k}_{2, \max }$ & $-2.59 \times 10^{-7}$ & $8.92 \times 10^{-5}$ & -0.0092 & 0.4205 & -4.9386 & 0.9999 \\
\hline $\mathrm{k}_{2, \min }$ & $1.97 \times 10^{-6}$ & -0.0006 & 0.0742 & -4.4980 & $\begin{array}{c}135.35 \\
-1611.33\end{array}$ & 0.9988 \\
\hline
\end{tabular}




\section{Discussion}

In this study, the mechanical behavior of the age-dependent aorta wall was investigated through an experimental and computational approach. To do this, the uniaxial tensile test for aorta wall tissue of porcine considering collagen fiber orientation was carried out, and the numerical experiment using HGO model-implemented ABAQUS was conducted to verify the experiments. After the validation of the simulation technique, the material characteristics of abdominal aorta wall of healthy humans were simulated, and the material parameters of HGO model under uniaxial tension were determined using polynomial regression equations in order to predict the uniaxial tensile behavior of the abdominal aorta wall of healthy humans.

According to the literature, aging has a significant effect on the structure and function of the arterial wall [31,32]. They studied the evolution of structural components for the human thoracic aorta from the strain energy function considering the composition of the soft tissue, and the axial stretch and residual strain in the biomechanical analysis were considered in order to examine the variation of the collagen fiber. In addition, the other research team studied the variation of the behavior of aortic wall according to age from the constitutive modeling approach. As a result, the age-related variation in pressure-load bearing from elastin to collagen was examined due to the decrease in elastin stiffness and the increase in collagen recruitment.

In this study, the increase of stretch and the decrease of stress and Young's modulus in the aortic tissue were investigated with the progress of age from the literature [17,31,32]. Moreover, the numerical simulations considering the variation rate of the material constants based on the age were conducted. In this simulation, the range of the material constants, which could simulate the behavior of the aortic tissue, were determined from the trend lines of the maximum and minimum values in the experiment results. The trend line of the minimum value could be applied to the study for aortic disease considering the stress and stretch in the severe condition.

In the experiments, the tensile test specimens were placed in saline at $38-39^{\circ} \mathrm{C}$, and the uniaxial tensile tests were performed within $12 \mathrm{~h}$ of harvest since it was important to maintain the environmental conditions in the experiment, such as temperature and elasticity. In other words, the environmental conditions in the tensile test should be maintained as the conditions in the porcine body since the material characteristics for soft tissue depend on the environmental conditions. In addition, the uniaxial tensile test specimens were generally produced as the dog-bone type; however, it is quite difficult to produce the specimens as the dog-bone type. Using the method obtained from the literature, the tensile test specimens were prepared by cutting the tissue into the dog-bone type without curvature [29]. This method had the disadvantage of inducing stress concentration in the center of the specimen, but it had the advantage of avoiding the stress concentration in the jig and reducing the failure rate of the experiment.

The preconditioning test was adopted to guarantee the straight configuration of the test specimen before the main test. In addition, the viscoelastic effect was not considered during the main test. In other words, a preconditioning test, involving 10 loading and unloading cycles of the specimen strip to $5 \%$ of its length at $4 \%$ of specimen length $/ \mathrm{min}(1 \mathrm{~mm} / \mathrm{min})$, was conducted. After preconditioning, the strip was uniaxially extended at $4 \%$ specimen length $/ \mathrm{min}(1 \mathrm{~mm} / \mathrm{min})$ until failure. In some articles, the displacement rate of $20 \%$ of specimen length/min was adopted during the uniaxial tensile test, for example Raghavan et al. [11], Ninomiya et al. [17], and Pierce et al. [30], to avoid the viscoelastic effect of tissue. However, the authors could not find any differences of the stress-stretch curves between $4 \%$ $(1 \mathrm{~mm} / \mathrm{min}$ or $0.0007 / \mathrm{s})$ and $20 \%(5 \mathrm{~mm} / \mathrm{min}$ or $0.003 / \mathrm{s})$ of specimen length/min through the comparison test. Accordingly, the displacement rate of $1 \mathrm{~mm} / \mathrm{min}$ was selected in this study.

On the other hand, in order to consider the viscoelastic effect during the experiment, a higher strain rate than the strain rate in this study should be applied to the test specimen, and the stress-increasing effects on the stress-stretch curve should be quantitatively investigated.

In the simulation, the C3D8RH-type hexahedral element, which is the eight-node linear brick, reduced integration with hourglass control, hybrid with constant pressure, was adopted in order to 
analyze the incompressible material behavior such as soft tissue and to avoid the volume strain locking problem [25].

There were some limitations in this study. First of all, during the identification of the material parameters in the HGO model, the biaxial tensile test of each aorta layer, namely intima, media, and adventitia, is recommended. However, it is not an easy task to prepare the test specimen of porcine and human tissues separate from each layer. Even if these specimens are ready, it is difficult to carry out the biaxial tensile test on these specimens $[23,33]$. For these reason, the uniaxial test was carried out by many researchers such as Holzapfel [33], Peyraut et al. [34], Skacel and Bursa [35], Hajhashemkhani and Hematiyan [36], Karimi et al. [37], Shazly et al. [38], and Latorre et al. [39]. In their research, the material parameters of the HGO model such as $C_{10}, k_{1}$, and $k_{2}$ were identified using the uniaxial test for each layer or unified layers of soft biological tissue including aorta wall tissue.

Despite the aforementioned studies, unfortunately, the test data according to age cannot be easily found in the literature. One of the well-studied research works considering age, as well as the uniaxial tensile test for human aorta wall was Ninomiya et al.'s article [17]. Accordingly, their test data referred to the authors' study.

The authors recognized the problem and limitation regarding this point. Hence, in the next paper, the biaxial, as well as the uniaxial test will be carried out, and the material parameters of the HGO model will be determined through the technique presented in the present study.

The micro-structural analysis of tissue for evaluation of fiber orientation was not carried out since the fiber orientation for intimal, medial, and adventitial strips referred to Holzapfel's article [26].

In addition, an optical method is also preferred to measure the strain of soft tissue, but there are some obstacles. One of the most difficult factors is that it is not easy to make a marking point on living tissue for strain measurement, and there was a limitation of the experimental apparatus setup such as the video-extensometer for gauge distance measurements due to the cost. Due to these limitations, the cross-head displacement of the universal testing machine was postulated to be equivalent to the stretch of soft tissue.

\section{Concluding Remarks}

This study examined the computational simulations of the samples extracted from porcine and healthy human abdominal aorta to investigate the material parameters as a function of age. In addition, the computational simulation results were compared with the uniaxial tensile test results of the porcine specimens to verify the reliability of the numerical simulation technique. The simulations based on age were conducted on circumferentially- and longitudinally-oriented strips, and formulae were proposed to determine the material parameters according to age. The results exhibited the following general trends:

- According to the uniaxial tensile test results with respect to the loading direction, the circumferentiallyoriented strip samples exhibited a maximum tensile strength value of $2.49 \mathrm{MPa}$, which was $18.3 \%$ higher than that for the longitudinally-oriented strip samples, based on the average value of the experimental results. Therefore, the uniaxial tensile stress and stretch in the arterial tissue were identified to be dependent on the fiber orientation.

- In addition, the failure stress and stretch were investigated as a function of age, and the material constants for age were calculated based on the maximum and minimum trend lines. The HGO model was applied to the numerical model for anisotropic hyper-elastic materials during the numerical simulations.

- In the parametric study, the formulae associated with the value of the material constant for the ages of 46-89 years were proposed, and the proportion variance in the dependent variable that was predictable from the independent variable was examined.

The results obtained in the present study are expected to be applied to study aortic disease, and it is possible to predict the behavior of the soft tissue in aortic disease based on the numerical simulation 
technique from this study. In addition, in further studies, the uniaxial and biaxial experiments for human aortic tissue will be conducted, and the precise experimental method for the viscoelastic effect will be proposed from the strain rate-dependent tensile test of soft tissue. Moreover, the study for human aortic tissue will be performed based on the experimental results and compared to the clinical data. The material constants for the HGO model will be calculated from the polynomial regression method.

Author Contributions: Conceptualization: U.H., C.-W.L., C.-S.L., and D.-M.R.; methodology: U.H., C.-W.L. C.-S.L., and D.-M.R.; software: J.-H.Y., C.-H.S., and D.-M.R.; validation: U.H., J.-H.Y., and C.-S.L.; formal analysis: C.-W.L. and C.-H.S.; investigation: U.H., J.-H.Y. C.-S.L., and D.-M.R.; writing, original draft preparation: U.H., C.-S.L., and D.-M.R.; writing, review and editing: U.H., C.-W.L., C.-S.L., and D.-M.R.

Funding: This research was supported by the Basic Science Research Program through the National Research Foundation of Korea (NRF) funded by the Ministry of Education (2018R1A6A3A01012664; 2018R1D1A1B07044567).

Conflicts of Interest: The authors declare no conflict of interest. The funders had no role in study design, data collection and analyses, writing of the manuscript, nor in the decision to publish the results.

\section{References}

1. Tortora, G.J. Principles of Human Anatomy; HarperCollins College: New York, NY, USA, 1995.

2. Tortora, G.J.; Grabowski, S.R. Principles of Anatomy and Physiology; HarperCollins College: New York, NY, USA, 1996.

3. Hole, J.W.; Koos, K.A. Human Anatomy; William C. Brown: Dubuque, Iowa, 1994.

4. Van De Graaff, K.M. Human Anatomy; WCB/McGraw-Hill: Boston, MA, USA, 1998.

5. Sobotta, J. Atlas van de Menselijke Anatomie; Bohn Stafleu van Loghum: Houten, Netherlands, 2006.

6. Tsamis, A.; Krawiec, J.T.; Vorp, D.A. Elastin and collagen fibre microstructure of the human aorta in ageing and disease: A review. J. R. Soc. Interface 2013, 10, 20121004. [CrossRef] [PubMed]

7. Saphirstein, R.J.; Gao, Y.Z.; Jensen, M.H.; Gallant, C.M.; Vetterkind, S.; Moore, J.R.; Morgan, K.G. The focal adhesion: A regulated component of aortic stiffness. PLoS ONE 2013, 8, e62461. [CrossRef]

8. Gao, Y.Z.; Saphirstein, R.J.; Yamin, R.; Suki, B.; Morgan, K.G. Aging impairs smooth muscle-mediated regulation of aortic stiffness: A defect in shock absorption function? Am. J. Physiol. Heart Circ. Physiol. 2014, 307, H1252-H1261. [CrossRef] [PubMed]

9. Hong, Z.; Reeves, K.J.; Sun, Z.; Li, Z.; Brown, N.J.; Meininger, G.A. Vascular smooth muscle cell stiffness and adhesion to collagen I modified by vasoactive agonists. PLoS ONE 2015, 10, e0119533. [CrossRef]

10. Leloup, A.J.; Van Hove, C.E.; Heykers, A.; Schrijvers, D.M.; De Meyer, G.R.; Fransen, P. Elastic and muscular arteries differ in structure, basal NO production and voltage-gated Ca2+-channels. Front. Physiol. 2015, 6, 375. [CrossRef] [PubMed]

11. Raghavan, M.L.; Webster, M.W.; Vorp, D.A. Ex vivo biomechanical behavior of abdominal aortic aneurysm: Assessment using a new mathematical model. Ann. Biomed. Eng. 1996, 24, 573-582. [CrossRef] [PubMed]

12. Wang, D.H.; Makaroun, M.; Webster, M.W.; Vorp, D.A. Mechanical properties and microstructure of intraluminal thrombus from abdominal aortic aneurysm. J. Biomech. Eng. 2001, 123, 536-539. [PubMed]

13. Tong, J.; Cohnert, T.; Holzapfel, G. Diameter-related variations of geometrical, mechanical, and mass fraction data in the anterior portion of abdominal aortic aneurysms. Eur. J. Vasc. Endovasc. Surg. 2015, 49, 262-270. [CrossRef]

14. Shahmansouri, N.; Alreshidan, M.; Emmott, A.; Lachapelle, K.; El-Hamamsy, I.; Cartier, R.; Leask, R.; Mongrain, R. Investigation on the regional loss factor and its anisotropy for aortic aneurysms. Materials 2016, 9, 867. [CrossRef]

15. Yang, T.; Chui, C.K.; Yu, R.Q.; Qin, J.; Chang, S.K. Quasi-linear viscoelastic modeling of arterial wall for surgical simulation. Int. J. Comput. Assist. Radiol. Surg. 2011, 6, 829-838. [CrossRef]

16. Horny, L.; Adamek, T.; Gultova, E.; Zitny, R.; Vesely, J.; Chlup, H.; Konvickova, S. Correlations between age, prestrain, diameter and atherosclerosis in the male abdominal aorta. J. Mech. Behav. Biomed. Mater. 2011, 4, 2128-2132. [CrossRef] [PubMed]

17. Ninomiya, O.H.; Monteiro, J.A.T.; de Lourdes Higuchi, M.; Puech-Leão, P.; de Luccia, N.; Raghavan, M.L.; da Silva, E.S. Biomechanical properties and microstructural analysis of the human nonaneurysmal aorta as a function of age, gender and location: An autopsy study. J. Vasc. Res. 2015, 52, 257-264. [CrossRef] [PubMed] 
18. Courtial, E.-J.; Fanton, L.; Orkisz, M.; Douek, P.; Huet, L.; Fulchiron, R. Hyper-viscoelastic behavior of healthy abdominal aorta. IRBM 2016, 37, 158-164. [CrossRef]

19. Laksari, K.; Shahmirzadi, D.; Acosta, C.J.; Konofagou, E. Energy-based constitutive modelling of local material properties of canine aortas. R. Soc. Open Sci. 2016, 3, 160365. [CrossRef] [PubMed]

20. Fung, Y.; Fronek, K.; Patitucci, P. Pseudoelasticity of arteries and the choice of its mathematical expression. Am. J. Physiol. Heart Circ. Physiol. 1979, 237, H620-H631. [CrossRef]

21. Humphrey, J.D. Mechanics of the arterial wall: Review and directions. Crit. Rev. Biomed. Eng. 1995, 23, 1-162. [CrossRef]

22. Holzapfel, G.A.; Gasser, T.C.; Ogden, R.W. A new constitutive framework for arterial wall mechanics and a comparative study of material models. J. Elast. Phys. Sci. Solids 2000, 61, 1-48.

23. Gasser, T.C.; Ogden, R.W.; Holzapfel, G.A. Hyperelastic modelling of arterial layers with distributed collagen fibre orientations. J. R. Soc. Interface 2005, 3, 15-35. [CrossRef]

24. Holzapfel, G.A.; Ogden, R.W. Constitutive modelling of arteries. Proc. R. Soc. Lond. A Math. Phys. Eng. Sci. 2010, 446, 1551-1597. [CrossRef]

25. ABAQUS. ABAQUS/Standard User's Manual; ABAQUS: Waltham, MA, USA, 2014.

26. Holzapfel, G. Arterial tissue in health and disease: Experimental data, collagen-based modeling and simulation, including aortic dissection. In Biomechanical Modelling at the Molecular, Cellular and Tissue Levels; Springer: Berlin/Heidelberg, Germany, 2009; pp. 259-344.

27. Spencer, A.J.M. Constitutive theory for strongly anisotropic solids. In Continuum Theory of the Mechanics of Fibre-Reinforced Composites; Springer: Berlin/Heidelberg, Germany, 1984; pp. 1-32.

28. Holzapfel, A.G. Nonlinear Solid Mechanics II; Wiley: Hoboken, NJ, USA, 2000.

29. Forsell, C.; Swedenborg, J.; Roy, J.; Gasser, T.C. The quasi-static failure properties of the abdominal aortic aneurysm wall estimated by a mixed experimental-numerical approach. Ann. Biomed. Eng. 2013, 41, 1554-1566. [CrossRef]

30. Pierce, D.M.; Maier, F.; Weisbecker, H.; Viertler, C.; Verbrugghe, P.; Famaey, N.; Fourneau, I.; Herijgers, P.; Holzapfel, G.A. Human thoracic and abdominal aortic aneurysmal tissues: Damage experiments, statistical analysis and constitutive modeling. J. Mech. Behav. Biomed. Mater. 2015, 41, 92-107. [CrossRef] [PubMed]

31. Zulliger, M.A.; Stergiopulos, N. Structural strain energy function applied to the ageing of the human aorta. J. Biomech. 2007, 40, 3061-3069. [CrossRef] [PubMed]

32. Spronck, B.; Heusinkveld, M.H.; Donders, W.P.; de Lepper, A.G.; Op't Roodt, J.; Kroon, A.A.; Delhaas, T.; Reesink, K.D. A constitutive modeling interpretation of the relationship among carotid artery stiffness, blood pressure, and age in hypertensive subjects. Am. J. Physiol. Heart Circ. Physiol. 2014, 308, H568-H582. [CrossRef] [PubMed]

33. Holzapfel, G.A. Determination of material models for arterial walls from uniaxial extension tests and histological structure. J. Theor. Biol. 2006, 238, 290-302. [CrossRef] [PubMed]

34. Peyraut, F.; Feng, Z.-Q.; Labed, N.; Renaud, C. A closed form solution for the uniaxial tension test of biological soft tissues. Int. J. Non-Linear Mech. 2010, 45, 535-541. [CrossRef]

35. Skacel, P.; Bursa, J. Comparison of constitutive models of arterial layers with distributed collagen fibre orientations. Acta Bioeng. Biomech. 2014, 16, 47-58. [PubMed]

36. Hajhashemkhani, M.; Hematiyan, M.R. Determination of material parameters of isotropic and anisotropic hyper-elastic materials using boundary measured data. J. Theor. Appl. Mech. 2015, 53, 895-910. [CrossRef]

37. Karimi, A.; Navidbakhsh, M.; Shojaei, A. A combination of histological analyses and uniaxial tensile tests to determine the material coefficients of the healthy and atherosclerotic human coronary arteries. Tissue Cell 2015, 47, 152-158. [CrossRef]

38. Shazly, T.; Rachev, A.; Lessner, S.; Argraves, W.S.; Ferdous, J.; Zhou, B.; Moreira, A.M.; Sutton, M. On the uniaxial ring test of tissue engineered constructs. Exp. Mech. 2015, 55, 41-51. [CrossRef]

39. Latorre, M.; Romero, X.; Montans, F.J. The relevance of transverse deformation effects in modeling soft biological tissues. Int. J. Solids Struct. 2016, 99, 57-70. [CrossRef]

(C) 2019 by the authors. Licensee MDPI, Basel, Switzerland. This article is an open access article distributed under the terms and conditions of the Creative Commons Attribution (CC BY) license (http://creativecommons.org/licenses/by/4.0/). 